\title{
HST emission line images of the Orion HII region: proper motions and possible variability
}

\section{Leonel Gutiérrez ${ }^{1,4}$, Corrado Giammanco ${ }^{2}$ and John E. Beckman ${ }^{1,3}$}

${ }^{1}$ Instituto de Astrofśsica de Canarias, La Laguna, Tenerife, Spain. email: jeb@iac.es

${ }^{2}$ Physikalisches Institut der Universität Bern, Switzerland. corrado.giammanco@space.unibe.ch

${ }^{3}$ Consejo Superior de Investigaciones Científicas, Spain.

${ }^{4}$ Universidad Nacional Autónoma de México, Ensenada, México. leonel@astrosen.unam.mx

Using HST emission line images of the Orion Nebula, separated by 7 years in epoch, we have obtained evidence of localized temporal variability of both density and temperature during this period. Applying a digital filter to reduce high frequency noise, we used images in $\mathrm{H} \alpha$ and [OIII] to quantify separately the variability in these two parameters. We detected fractional temperature variations of order $0.4 \%$ on scales of $2 \times 10^{-2} \mathrm{pc}$. The same images yielded proper motion information; using cross-correlation to optimize the accuracy of the differential measurements we produced velocity field maps across the nebula, with vectors ranging up to $\sim 130 \mathrm{~km} \mathrm{~s}^{-1}$ across the line of sight. It is notable that in zones of rapid proper motion we find by far the largest density variations, as would be expected. It is much easier to quantify the temperature variations, on the other hand, in zones with low or zero detectable proper motion (see the other figure here), though these temperature variations appear across the whole face of the nebula.
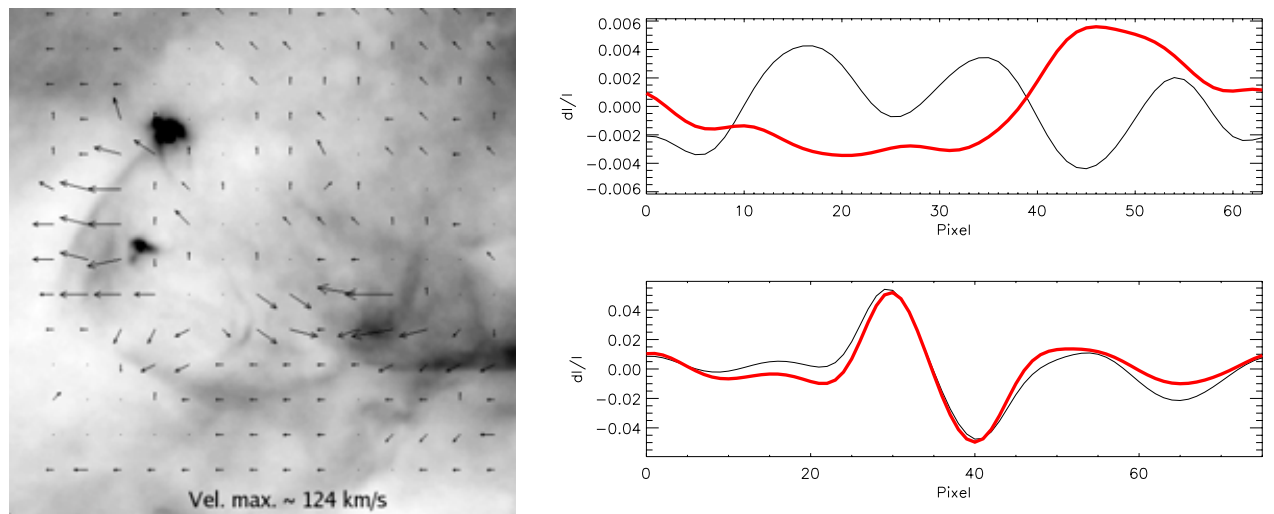

Left: Proper motion velocity vector map superposed on a portion of the $\mathrm{H} \alpha$ image. The map presented covers an area of 0.18 x 0.18 parsec. Right - Upper panel: Scan across a kinematically quiescent field. The changes in surface brightness in $\mathrm{H} \alpha$ (red, thick line) and [OIII] (black, thin line) are essentially in antiphase, as predicted for changes produced by localized temperature variations between the two epochs with only small density changes. Lower panel: Scan across a part of the nebula where rapid movement is detected. The changes in $\mathrm{H} \alpha$ and [OIII] surface brightness are in phase as predicted for zones affected by density variations. We can use plots of these kinds to separate the changes in the two variables.

\section{References}

Corrado, G., Beckman, J.E. \& Gutiérrez, L. 2006, in preparation

O'Dell, C.R., Peimbert, M. \& Peimbert, A. 2003, ApJ 125, 2590

Peimbert, M. 1967, ApJ 150, 825 